\title{
Low Metabolism and Inactive Lifestyle of a Tropical Rain Forest Bird Investigated via Heart-Rate Telemetry
}

\author{
Silke S. Steiger ${ }^{1, \star}$ \\ J. Patrick Kelley ${ }^{1, \dagger}$ \\ William W. Cochran ${ }^{2}$ \\ Martin Wikelski ${ }^{1,3, \#}$ \\ ${ }^{1}$ Department of Ecology and Evolutionary Biology, Guyot \\ Hall 403, Princeton University, Princeton, New Jersey 08540; \\ ${ }^{2}$ Illinois Natural History Survey, Peabody Drive, Champaign, \\ Illinois 61821; ${ }^{3} \mathrm{Max}$ Planck Institute for Ornithology, \\ Department of Migration and Immuno-Ecology, 78315 \\ Radolfzell, Germany
}

\section{Introduction}

Nighttime resting metabolic rate (RMR) is generally lower in tropical birds than in comparable temperate-zone or arctic birds (e.g., Vleck and Vleck 1979; Hails 1983; Klaassen 1995; Lindstrom and Klaassen 2003; Tieleman et al. 2006; Wiersma et al. 2007). Metabolic rates are even lower in tropical populations within the same species when birds are raised under similar captive conditions, suggesting intrinsic differences in energy metabolism between birds living in different habitats/ latitudes (Klaassen 1995; Furness 2003; Wikelski et al. 2003; Tieleman 2007). In addition, there is laboratory evidence that tropical birds may use facultative and regulated hypothermia and torpor to save energy (e.g., Bartholomew et al. 1983; Merola-Zwartjes and Ligon 2000). Some authors have also suggested that tropical birds lead a much more "leisurely" lifestyle than other birds (Hamilton 1966; Ricklefs 1991; Wikelski et al. 2003) and exhibit lower activity rates (Weathers and Stiles 1989; Martin 1996; Wikelski and Ricklefs 2001), consistent with low rates of energy expenditure.

In this study, we used heart rate to estimate the nighttime RMR, daily energy expenditure (DEE), and locomotor activity of a small, long-lived, rain forest suboscine passerine, the spotted antbird (Hylophylax naevioides; family Thamnophilidae; del Hoyo et al. 2003). These small ( 17-g) insectivorous birds are characterized by life spans longer than $15 \mathrm{yr}$ and high annual survival rates of up to $81 \%$ (Willis 1972, 1983; Brawn et al. 1995; Hau et al. 2000). Spotted antbirds are members of mixed bird aggregations that often follow army ant swarms to catch insects flushed by the ants (Willis 1972). On average, about $50 \%$ of their food is taken at ant swarms (Willis 1972). Spotted antbirds generally remain in the undergrowth of the tropical rain forest (below $2 \mathrm{~m}$ height), where they hop from perch to perch and rarely fly more than $20 \mathrm{~m}$ at a time (Willis 1972). This niche is adopted by only $2 \%-3 \%$ of the forest songbirds at any one site and only about $10 \%$ of the family (Ridgely and Gwynne 1989). Spotted antbirds exhibit an unusually sedentary lifestyle that makes them likely to be among the more inactive species in a community. Thus, we hypothesized that Neotropical spotted antbirds show reduced rates of energy turnover and low activity in the wild. Combined measurements of nighttime RMR and DEE of, for example, small free-living tropical songbirds are rare (Ricklefs et al. 1996; Tieleman et al. 2006).

Several studies have shown that heart rate is a useful indicator of energy expenditure in free-ranging animals and perhaps as accurate as the doubly labeled water (DLW) method (Nolet et al. 1992; Bevan et al. 1995; Shaffer et al. 2001; Butler et al.
Konstanzer Online-Publikations-System (KOPS)

URN: http://nbn-resolving.de/urn:nbn:de:bsz:352-opus-108243

URL: http://kops.ub.uni-konstanz.de/volltexte/2010/10824/ 
2004; Cooke et al. 2004; Cochran and Wikelski 2005; Bowlin and Wikelski 2008). Heart rate allows for the prediction of oxygen consumption and thus energy expenditure via Fick's equation (Bevan et al. 1994; Green et al. 2001; Butler et al. 2004). However, calibration experiments are needed to determine the exact relationship between the two variables. Heart rate studies have generally been conducted on large vertebrates because of the previous lack of techniques to monitor heart rate in small animals (Bevan et al. 1995; Shaffer et al. 2001; Green et al. 2002; Cooke et al. 2004).

Our study had three main aims: (i) to test whether captive spotted antbirds are able to reduce body temperature to save energy, (ii) to estimate the nighttime RMR and DEE of freeliving spotted antbirds via heart rate telemetry, and (iii) to compare the estimated values of energy expenditure in the wild with general predictions of nighttime RMR and DEE in temperate-zone and arctic birds of similar size.

\section{Material and Methods}

Animals and Study Site

Twenty male spotted antbirds were captured in mist nets on Barro Colorado Island, Panama $\left(9^{\circ} \mathrm{N}, 79^{\circ} \mathrm{W}\right)$, from March 24 to April 7, 2002, September 18 to October 17, 2002, and March 24 to April 7, 2003. On Barro Colorado Island, the average annual temperature is $27^{\circ} \mathrm{C}$ (with a diurnal temperature range of $9^{\circ} \mathrm{C}$; Dietrich et al. 1982). Birds were attracted to nets with song playbacks broadcast using a tape recorder and loudspeakers (Wikelski et al. 1999). Birds were carried to the laboratory in cloth bags and weighed to the nearest $0.2 \mathrm{~g}$ with spring balances. We believe that we monitored only nonbreeding birds, for the following reasons. First, we never observed any courtship feeding or copulation. Second, we never recorded any nestbuilding behavior. Third, we never monitored incubating birds. Fourth, we never saw any fledglings close to the observed birds.

\section{Respirometry}

We measured rates of oxygen consumption $\left(\dot{\mathrm{V}}_{2}\right)$ and carbon dioxide production $\left(\dot{\mathrm{V}}_{\mathrm{CO}_{2}}\right)$ in an open-flow, push-through respirometry system. External, naturally humid air (85\%-95\% humidity, $\sim 25^{\circ} \mathrm{C}$ ) was pumped through a mass flow controller (TR-FCI, Sable Systems, Las Vegas, NV) and a multiplexer (V20 , Sable Systems) into the metabolic and reference chambers. Flow rate was $1,000 \mathrm{~mL} / \mathrm{min}$, and the flow controller was calibrated before use via a bubble meter. A factory calibration after use indicated that flow rate errors were less then $1.2 \%$. Air leaving the chambers was dehumidified using a PeltierEffect Condenser (PC-1; Sable Systems), and $\mathrm{CO}_{2}$ concentration was measured from a subsample of the outlet flow (CA1B, Sable Systems). Before $\dot{\mathrm{V}}_{2}$ was determined (FC-1B, Sable Systems), Drierite was used to scrub potential remaining water from the air. The respirometry system was tested for leaks. No loss of air was observed.
We estimated instantaneous oxygen consumption from the equation of Bartholomew et al. (1981):

$$
\mathrm{FEo}_{2}(\mathrm{eq})=\mathrm{FEO}_{2}(t-1)+\left[\frac{\mathrm{FEo}_{2}(t)-\mathrm{FEo}_{2}(t-1)}{1-e^{(-\dot{V} / V) \Delta t}}\right],
$$

where $\mathrm{FEO}_{2}$ is the oxygen concentration in the excurrent air, $\mathrm{FEO}_{2}(\mathrm{eq})$ is the equilibrium value, $V$ is the volume of the system including tubing, $\dot{V}$ is the flow rate through the system, and $\Delta t$ is the interval between measurements at times $t$ and $t-1$. We determined the denominator of this equation (the so-called $Z$ value) empirically using Datacan (Sable Systems). We then calculated the rate of oxygen consumption with equation $(3 b)$ of Withers (1977):

$$
\dot{\mathrm{V}} \mathrm{O}_{2}=\dot{V}\left[\frac{\left(\mathrm{FIo}_{2}-\mathrm{FEO}_{2}\right)-\dot{\mathrm{V}} \mathrm{CO}_{2} \times \mathrm{FIo}_{2}}{1-\mathrm{FIo}_{2}}\right],
$$

where $\mathrm{FIO}_{2}$ is the incurrent oxygen concentration. We also determined the respiratory quotient (RQ) - the ratio of $\mathrm{CO}_{2}$ produced to $\mathrm{O}_{2}$ consumed-and used it to calculate thermal equivalents and metabolic rate (in $\mathrm{kJ} / \mathrm{d}$, following Walsberg and Hoffman 2005).

\section{Influence of Ambient Temperature on Oxygen Consumption and Body Temperature}

To determine the relationship between ambient temperature, metabolic rate, and body temperature, we measured the oxygen consumption of eight males in 1-L chambers for $4 \mathrm{~h}$ after 2100 hours (i.e., starting $3 \mathrm{~h}$ after sunset) between March 24 and April 7, 2002. Birds were fed ad lib. during the day. At sunset ( $\sim 1800$ hours), the food was removed from the cage. Thermocouples in the respirometry chamber and thermocouples inserted deep $(\sim 1 \mathrm{~cm})$ into the birds' cloacae and superglued to cloacal feathers (Ricklefs and Williams 2003) allowed for continuous readings of ambient and body temperatures, respectively. We used the "up-down" and "down-up" temperature protocol developed by Ricklefs and Williams (2003). In short, birds were introduced into the chamber at an ambient temperature of $29^{\circ} \mathrm{C}$ and allowed to settle for $1 \mathrm{~h}$. Then we determined the lowest 3-min average $\dot{\mathrm{V}}_{2}$ and $\dot{\mathrm{V}}_{\mathrm{CO}_{2}}$ during the following $30 \mathrm{~min}$. Subsequently, we either slowly decreased (down to $14^{\circ} \mathrm{C}$ ) or increased (up to $36^{\circ} \mathrm{C}$ ) the ambient temperature in random sequence. Each up-down or down-up temperature protocol lasted for $3 \mathrm{~h}$. During this protocol, we averaged values of oxygen consumption for windows of $3 \mathrm{~min}$ whenever ambient temperatures in the chambers had changed by $2^{\circ} \mathrm{C}$ (Fig. 1 ).

\section{Calibration of Heart Rate versus Oxygen Consumption}

The relationships between energy expenditure and heart rate were determined between March 24 and April 9, 2003, in six males. Birds with heart rate transmitters (for attachment of heart rate 


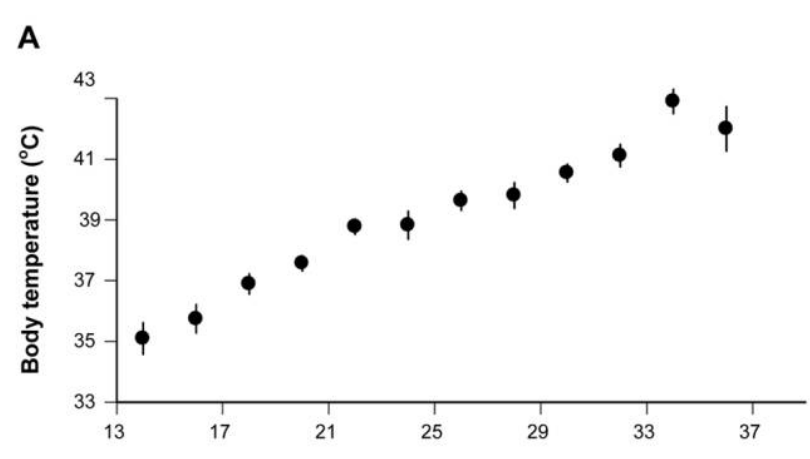

B

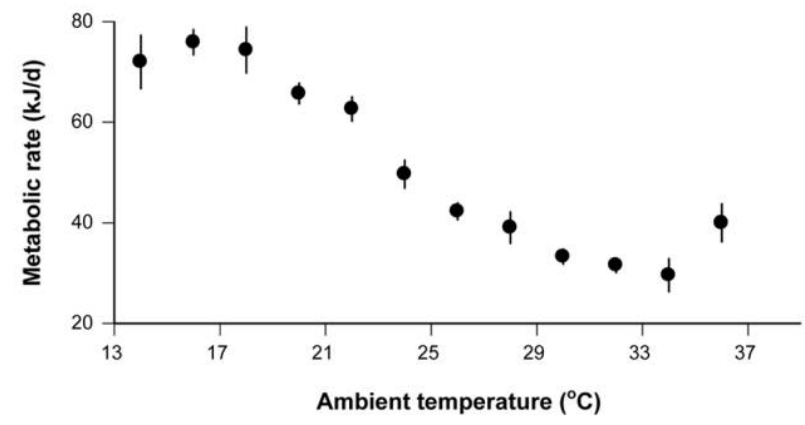

Figure 1. Relationship between ambient temperature and body temperature $(A)$ and metabolic rate $(B)$ as determined in laboratory respirometry trials. Data show means \pm SE for eight male spotted antbirds. Note that body temperature decreases linearly with ambient temperature even within the ambient temperature range where energy expenditure is minimal $\left(30^{\circ}-34^{\circ} \mathrm{C}\right)$.

transmitters, see below) were placed in 7-L plastic metabolic chambers, and heart rate, $\dot{\mathrm{V}}_{2}$, and $\dot{\mathrm{V}}_{\mathrm{CO}_{2}}$ were measured continuously for a period of $38.8 \pm 5.6 \mathrm{~h}$ (mean $\pm \mathrm{SE}$ ) at an ambient temperature of $25.5^{\circ} \pm 2{ }^{\circ} \mathrm{C}$ (mean $\pm \mathrm{SD}$ ). Birds could move and hop freely and had mealworms and water ad lib. In order not to add unnecessary stress during these measurements, we did not use a smaller metabolic chamber and a higher flow rate (M. Wikelski, unpublished data). To account for the washout time for the chamber and for analyzer responses, instantaneous oxygen consumption was calculated as described above after adjusting flow rate for the amount of water due to humidity (LabAnalyst X, Riverside, CA; Withers 2001).

Heart rate was recorded as a sound file in a laptop computer. After measurements, the transmitters were removed and birds were weighed and then released at their original capture sites. $\dot{\mathrm{V}}_{2}$ and heart rate data were obtained from each bird for nine random time points, when $\dot{\mathrm{V}}_{\mathrm{CO}_{2}}$ was stable $( \pm 1 \mathrm{~mL} / \mathrm{h})$ for on average $121 \pm 69 \mathrm{~s}$ (mean $\pm \mathrm{SD}$; range: $37-375 \mathrm{~s}$ ). For one spotted antbird we obtained only four time points. Instantaneous oxygen consumption and heart rate were averaged over these periods.

\section{Heart Rate Transmitter and Attachment}

We used newly developed $\sim 1$-g heart rate transmitters $(0.2$ $\mathrm{cm} \times 0.7 \mathrm{~cm} \times 0.3 \mathrm{~cm} ; \mathrm{SP} 2000 \mathrm{HR}$, Sparrow Systems, Fisher,
IL) that emitted a continuous signal amplitude (AM) that was frequency modulated by heart muscle potentials (FM; for detailed technical information, see Cochran and Wikelski 2005). Transmitters had a 9-cm flexible wire antenna that was $0.2 \mathrm{~mm}$ in diameter. Transmitters had a reception range of at least 100 $m$ even in dense tropical forest. In short, birds were anesthetized with a mixture of Isoflurane (Abbott Laboratories, North Chicago, IL) and air. A patch of approximately $2 \mathrm{~cm}^{2}$ of back body plumage close to the birds' center of gravity was cut to the feather stumps (following Raim 1978). The heart rate transmitter was glued to a $<2-\mathrm{cm}^{2}$ cloth. The cloth was then glued onto the feather stumps on the back of the bird with eyelash adhesive. Two wire electrodes were placed under the skin (for a detailed description of the attachment procedure, see Cochran and Wikelski 2005). Birds were released within $1 \mathrm{~h}$, and we could not detect an apparent change in the behavior of radiotagged birds: they foraged, defended their territory, and continued to interact socially after their release.

We are aware that despite their small size, the heart rate transmitters are somewhat heavy ( $5 \%$ body mass) for spotted antbirds. To quantitatively assess potential behavioral differences in birds fitted with transmitters, we observed the prey capture rate in each of six spotted antbirds with and without regular transmitters at an army ant swarm for a total of $2 \mathrm{~h}$ each. We observed the focal bird (with transmitter) for $2 \mathrm{~min}$ continuously and then immediately switched observations to a nonmanipulated conspecific for the same amount of time, treating these observations statistically as a matched pair. We could not detect differences in the number of prey items captured (Wilcoxon test, $N=6, P=0.4$ ) or in the total number of moves (Wilcoxon test, $N=6, P=0.65$ ). Note, however, that the transmitters could potentially also affect flight dynamics. Furthermore, feather clipping for transmitter attachment may affect conductance, but at present we are unable to quantify these effects in the wild.

Heart Rate Telemetry and Analysis of Field Energy Expenditure

The heart rates of six free-ranging birds were recorded for at least $24 \mathrm{~h}$ continuously during fieldwork conducted between September 18 and October 19, 2002. Average ambient temperature during day and night was $27.2^{\circ} \pm 0.11^{\circ} \mathrm{C}$ (minimum: $23^{\circ} \mathrm{C}$; maximum: $32.4^{\circ} \mathrm{C}$ ) and $24.4^{\circ} \pm 0.04^{\circ} \mathrm{C}$ (minimum: $22.3^{\circ} \mathrm{C}$; maximum: $26.6^{\circ} \mathrm{C}$ ), respectively. A receiver (AR8200, AOR, Tokyo) was used to recover the continuous transmitter signal. The transmitted acoustic signal was recorded on a minidisc (MZ-S1, Sony) and later transferred to a computer and analyzed with Cool Edit 2000 software (Syntrillium Software, Phoenix, AZ; as described in Cochran and Wikelski 2005). Every $5 \mathrm{~min}$ throughout $24 \mathrm{~h}$ we determined instantaneous heart rate from an average of 10 beats. We obtained, on average, $243 \pm 13$ measurements per day per bird in a 24 -h period (of 288 possible; Table 1 ). In addition, for one bird, every heartbeat was counted throughout $24 \mathrm{~h}$, and these values were averaged per minute. These results were only $1.94 \%$ different from the 5-min average values of the same bird. 
Table 1: Identification number (ID), mass, number of counted 5-min blocks, mean heart rates (day, night, 24-h period), and estimated daily energy expenditure (DEE) with $95 \%$ confidence intervals (CI) obtained for six male spotted antbirds on Barro Colorado Island, Panama

\begin{tabular}{|c|c|c|c|c|c|c|c|c|}
\hline \multirow[b]{2}{*}{ ID } & \multirow{2}{*}{$\begin{array}{l}\text { Mass } \\
(\mathrm{g})\end{array}$} & \multirow{2}{*}{$\begin{array}{l}5 \text {-min } \\
\text { Block }(N)\end{array}$} & \multicolumn{3}{|c|}{ Heart Rate (beats/min \pm SE) } & \multicolumn{3}{|c|}{$\mathrm{DEE}(\mathrm{kJ} / \mathrm{d})$} \\
\hline & & & Day & Night & 24-h Period & Estimate & Lower 95\% CI & Upper $95 \%$ CI \\
\hline 1 & 18.25 & 277 & $515.7 \pm 8.5$ & $322.0 \pm 4.0$ & $418.8 \pm 7.5$ & 28.34 & 23.73 & 32.99 \\
\hline 2 & 18.10 & 196 & $642.3 \pm 6.6$ & $367.8 \pm 4.9$ & $488.3 \pm 10.5$ & 35.16 & 30.52 & 39.80 \\
\hline 3 & 16.50 & 233 & $571.9 \pm 7.9$ & $423.8 \pm 4.6$ & $489.3 \pm 6.5$ & 41.25 & 36.62 & 45.88 \\
\hline 4 & 17.50 & 253 & $614.1 \pm 4.8$ & $380.1 \pm 5.8$ & $496.7 \pm 8.3$ & 38.16 & 32.17 & 42.78 \\
\hline 5 & 18.25 & 219 & $579.0 \pm 5.1$ & $395.4 \pm 5.6$ & $512.8 \pm 7.1$ & 36.80 & 32.16 & 41.44 \\
\hline 6 & 17.00 & 277 & $685.4 \pm 6.3$ & $416.0 \pm 5.6$ & $545.4 \pm 9.1$ & 44.42 & 39.80 & 49.05 \\
\hline Mean & 17.60 & 242.5 & $601.4 \pm 24.2$ & $384.2 \pm 15.1$ & $491.9 \pm 11.6$ & 37.36 & 32.73 & 41.98 \\
\hline
\end{tabular}

We observed locomotor activity continuously, either directly, when the bird was in sight, or indirectly, via a clearly audible sudden increase of heart rate that occurred only when a flight or hop was performed. We visually confirmed that locomotor movements and sudden increases in heart rate always coincided in time. Minor locomotor movements were not analyzed (e.g., preening, bathing, posture changes related to stationary foraging). One-minute periods were analyzed to quantify locomotor activity.

We used heart rate data to estimate each bird's metabolic rate by applying a multiple-regression equation (including mass) derived from the calibration procedure. The lowest heart rate value measured per night was used to estimate RMR in the field. RMR results were compared with values predicted by Aschoff and Pohl (1970), Bennett and Harvey (1987), Reynolds and Lee (1996), and McKechnie and Wolf (2004). Note that Bennett and Harvey (1987) and McKechnie and Wolf (2004) included only data that met the criteria for basal metabolic rate. We estimated DEE by calculating energy expenditure from instantaneous heart rate measurements and averaged those values over $24 \mathrm{~h}$. DEE results were compared with values predicted for small birds (Nagy et al. 1999).

\section{Statistical Analysis}

It should be noted that, as for the DLW method, large individual errors might arise when the heart rate method is used (Butler et al. 2004). Thus, averaging larger sample sizes will be more informative, and that is a procedure we follow here. Data were analyzed with SPSS (ver. 14.0, SPSS, Chicago). Two-tailed tests were used, and results are presented as means $\pm 1 \mathrm{SE}$, unless otherwise stated. We used least squares regressions to determine the relationship between heart rate and metabolic rate and ANCOVA to test whether slopes and intercepts were significantly different between individuals. Slopes were not different, but intercepts were; thus, we included the intercept as a random effect (Green et al. 2001) in a linear mixed model. For the final model, we included mass as a covariate. We separated daytime from nighttime as the period between the first locomotor activity in the morning and the last locomotor activity in the evening.

\section{Ethical Note}

This study was approved by the Princeton Institutional Animal Care and Use Committee (protocol 1428). The treatment of the animals adheres to the American Ornithologists' Union recommendations for the use of wild animals in research.

\section{Results}

Relationship among Ambient Temperature, Metabolic Rate, and Body Temperature

Birds in the respirometry chambers had high body temperatures $\left(\sim 40^{\circ} \mathrm{C}\right)$ at ambient temperatures of $30^{\circ} \mathrm{C}$ (Fig. 1). Although individuals increased their metabolic rate at decreasing ambient temperatures below $30^{\circ} \mathrm{C}$, their body temperatures steadily decreased, suggesting that spotted antbirds have limited ability to cope physiologically with cold. The decrease in body temperature and increase in metabolic rate at low temperatures may also indicate poor insulation in spotted antbirds. A similar pattern has been observed in the tropical variable seedeater (Sporophila aurita; Weathers 1997). Below an ambient temperature of $\sim 18^{\circ} \mathrm{C}$, birds did not increase energy expenditure any longer; thus, trials were stopped at ambient temperatures of $\sim 14^{\circ} \mathrm{C}$. Birds also increased body temperatures when warmed toward higher ambient temperatures. Above an ambient temperature of $\sim 34^{\circ} \mathrm{C}$, individuals increased energy expenditure again, which appeared to stabilize body temperature at $\sim 42^{\circ} \mathrm{C}$. Ambient temperature was not increased beyond $36^{\circ} \mathrm{C}$ to avoid undue stress on the birds. It appears that the spotted antbirds had only a very narrow thermoneutral zone (TNZ) where energy expenditure was stable at changing temperatures (ambient temperature range from approximately $30^{\circ}$ to $34^{\circ} \mathrm{C}$ ).

\section{Relationship between Metabolic Rate and Heart Rate}

The mean mass of birds used for calibration was $16.6 \pm 0.6 \mathrm{~g}$ before and $16.0 \pm 0.3 \mathrm{~g}$ after calibration (Table $2 ; t=1.0$, $P=0.3$ ). The mean mass of birds used for heart rate telemetry was $17.6 \pm 0.3 \mathrm{~g}$. On average, the RQ was $0.69 \pm 0.01$. Note that although the RQ usually is expected to be between 0.7 and 1 , there is evidence for values beyond that range in several bird 
Table 2: Individual regression equation variables for metabolic rates against heart rate for six male spotted antbirds from Barro Colorado Island, Panama

\begin{tabular}{|c|c|c|c|c|c|c|}
\hline \multirow[b]{2}{*}{$\mathrm{ID}^{\mathrm{a}}$} & \multirow[b]{2}{*}{ Mass (g) } & \multirow[b]{2}{*}{$N^{\mathrm{b}}$} & \multicolumn{4}{|l|}{ Variable } \\
\hline & & & $a^{c}$ & $b^{\mathrm{d}}$ & $r^{2}$ & $P$ \\
\hline 1 & 14.75 & 4 & -29.18 & .26 & .80 & .106 \\
\hline 2 & 17.00 & 9 & 6.62 & .15 & .65 & .009 \\
\hline 3 & 18.25 & 9 & -13.48 & .19 & .76 & .002 \\
\hline 4 & 17.00 & 9 & .45 & .14 & .54 & .025 \\
\hline 5 & 17.50 & 9 & -28.54 & .22 & .78 & .002 \\
\hline 6 & 15.25 & 9 & -19.67 & .28 & .81 & $<.001$ \\
\hline
\end{tabular}

species (Nolet et al. 1992; Walsberg and Wolf 1995; Hawkins et al. 2000). The maximum values for heart rate and $\dot{\mathrm{V}}_{2}$ were 695 beats/min and $125 \mathrm{~mL} / \mathrm{h}$, respectively. A significant linear relationship between heart rate and metabolic rate was found for every bird $\left(0.54<r^{2}<0.81\right.$; Fig. 2 ; Table 2$)$. The regression slopes were not significantly different among birds, but the intercepts were (ANCOVA: $F_{5,42}=7.7, P<0.001$ ). Thus, following Green et al. (2001), we adopted a random-effect model and included the intercept as a random factor and the slope as a fixed effect. We derived the following equation:

$$
\mathrm{MR}=0.09 f_{\mathrm{H}}-4.04,
$$

where MR is metabolic rate in $\mathrm{kJ} / \mathrm{d}$ and $f_{\mathrm{H}}$ is heart rate in beats per minute. After mass was included, we obtained the following relationship:

$$
\mathrm{MR}=59.07+0.09 f_{\mathrm{H}}-3.749 m,
$$

where $m$ is mass in grams. Using equation (11) from Green et al. (2001), we calculated the SE of the estimated MR (Table 1). The error associated with the variation between spotted antbirds $\left(d^{2}\right)$ was 13.25; the error associated with the scatter around the regression lines $\left(e^{2}\right)$ was 35.22 .

\section{Heart Rates of Free-Living Birds}

During the day, heart rate was 36\% higher and was also more variable than nocturnal heart rate (Fig. 3). A sharp increase in heart rate was detected from 0500 to 0700 hours, and a sharp decrease in heart rate was detected from 1700 to 1800 hours (Fig. 3). Heart rate started to increase in the early morning before the birds started to be active. The average nocturnal heart rate was $384.2 \pm 15.1$ beats/min, and the average diurnal heart rate was $601.4 \pm 24$ beats/min (Table 1 ). The mean daily heart rate $(24 \mathrm{~h})$ was $491.9 \pm 11.6$ beats $/ \mathrm{min}$. The maximum recorded heart rate, recorded during the day, was 824 beats/ min, which was 3.2 times as high as the lowest observed value, recorded at night, of 260 beats/min. Notably, $96.2 \%$ of the heart rates monitored in free-living spotted antbirds were within the range of heart rates measured during calibration. The heart rate frequency distribution was bimodal, with peaks occurring at 650 beats/min and at 400 beats/min; day $(N=730)$ and night values $(N=725)$ partially overlapped (Fig. $4 A)$.

\section{Locomotor Activity}

Locomotor activity started, on average, $16.3 \pm 2.3 \mathrm{~min}$ before official sunrise (which was at 0542-0550 hours) and ended $9.3 \pm 5.7 \mathrm{~min}$ after official sunset (which was at 1748-1757 hours). When active, spotted antbirds mostly hopped and never flew for more than ca. $20 \mathrm{~m}$ at any one time, thus, flying and hopping were combined into one measure of locomotor activity (moves/min). Note that the average movement rate is an average of the locomotor activity of only the active period (from $\sim 0550$ until $\sim 1750$ hours; see above). Locomotor activity was not normally distributed but highly skewed (Fig. $4 B$ ). During approximately $35 \%$ of the day, spotted antbirds were not locomotively active (no moves per minute; Fig. 4B). Diurnal locomotor activity was consistently low and stable throughout the day. On average, spotted antbirds moved $1.6 \pm 0.04$ times/ min throughout the day.

\section{Oxygen Consumption and Metabolic Rate of Wild Birds}

The calculated nighttime RMR for all six free-living birds was $20.1 \pm 1.9 \mathrm{~kJ} / \mathrm{d}$. Compared with the nighttime RMR predicted by Aschoff and Pohl (1970) and Reynolds and Lee (1996), our value for wild spotted antbirds was $21.3 \%$ (Fig. $5 A ; t=2.9$, $P<0.05$ ) and $22.9 \%$ (Fig. $5 A ; t=3.16, P=0.01$ ) lower than expected, respectively. However, compared with values predicted by Bennett and Harvey (1987) and McKechnie and Wolf (2004), observed RMRs for wild spotted antbirds were $30.2 \%$ (Fig. 5A; $t=-2.5, P<0.05$ ) and $27.2 \%$ (Fig. 5A; $t=-2.3$, $P<0.05)$ higher, respectively. Note that these studies predicted metabolic rate for captive, postabsorptive animals in a thermoneutral environment, whereas our wild study birds most

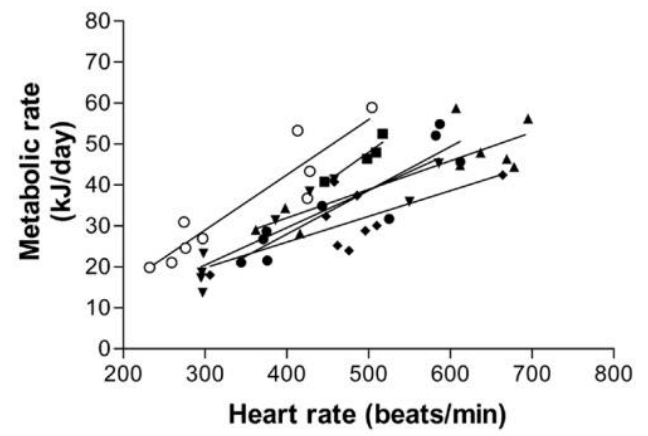

Figure 2. Linear relationships between heart rate (beats/min) and metabolic rate $(\mathrm{kJ} / \mathrm{d})$ for six male spotted antbirds (SPAN) from Barro Colorado Island, Panama: SPAN 1 (squares), SPAN 2 (triangles), SPAN 3 (inverted triangles), SPAN 4 (diamonds), SPAN 5 (filled circles) and SPAN 6 (open circles). The slopes are not significantly different, but the intercepts are. 

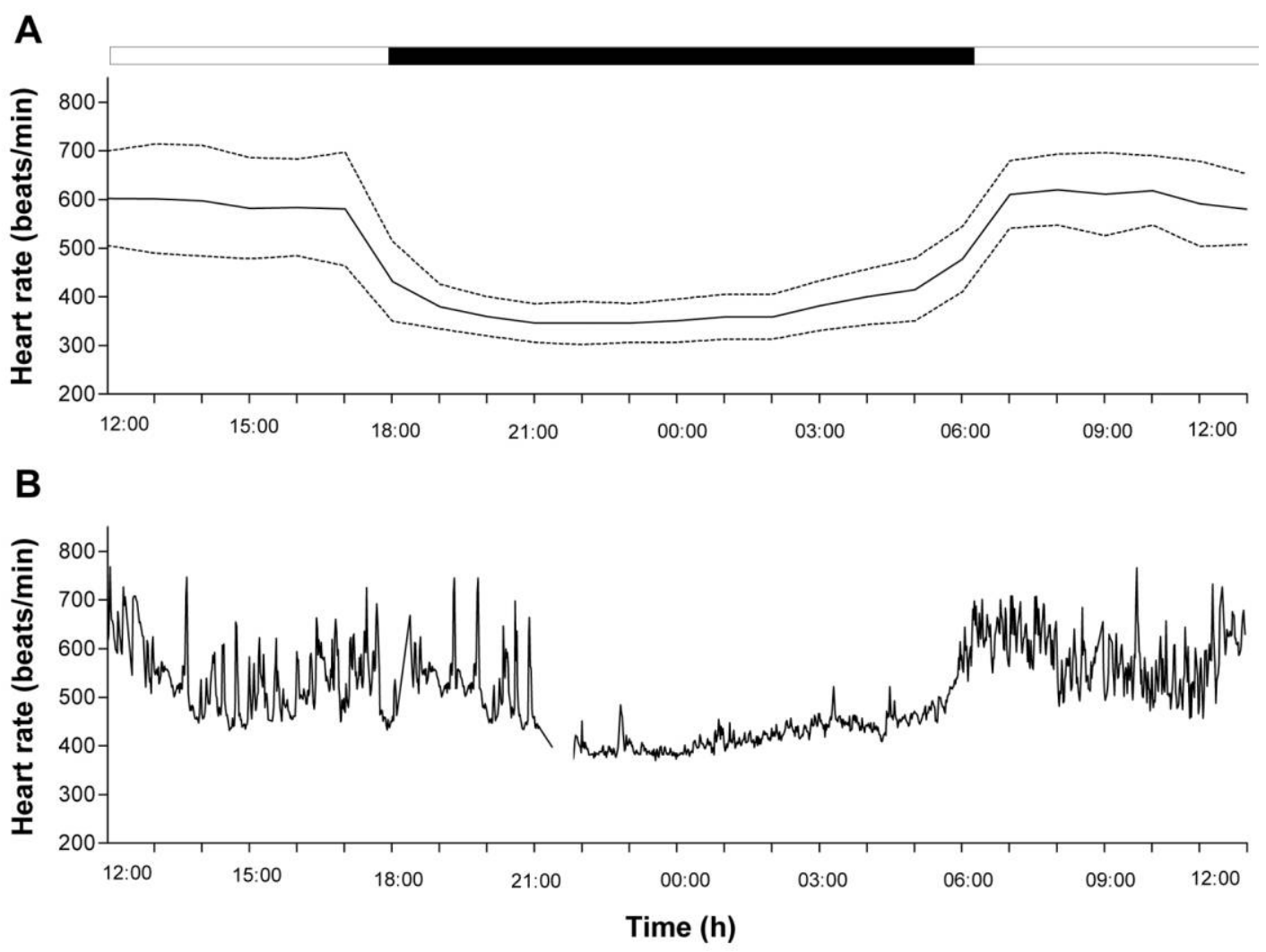

Figure 3. Daily heart rate patterns (beats/min). The black bar at the top indicates nighttime (inactive period), and the white bars indicate daytime. A, Mean hourly heart rates of six spotted antbirds. The solid line represents the hourly mean and the dashed lines \pm 1 SD. B, Secondby-second heart rate analysis conducted for spotted antbird 3. Heart rate was measured every second, and measurements were averaged every minute $(N=1,317)$.

likely did not experience thermoneutral conditions. Moreover, the birds in the wild may have been feeding. Thus, our values might be an overestimate of nighttime RMR, and therefore it is not surprising that our nighttime RMR values are higher than values predicted by Bennett and Harvey (1987) and McKechnie and Wolf (2004). DEE was $37.36 \pm 2.3 \mathrm{~kJ} / \mathrm{d}$. When we compared our estimate of DEE with Nagy's allometric equation (Nagy et al. 1999) for temperate-forest/small birds, spotted antbirds exhibited only $51 \%$ of the expected value (Fig. $5 B$; $t=15.1 ; P<0.001)$.

\section{Discussion}

Using heart rate telemetry, we measured both the nighttime RMR and the DEE of a small tropical passerine living in the rain forest understory. Before this study, the DEE of free-living tropical rain forest passerines had not been measured, perhaps because of the difficulty of recapturing such birds after a 24$\mathrm{h}$ period, making DLW measurements hard to conduct in the rain forest (Speakman 1998).

Our data show that the DEE is low in the wild compared with expected values for birds of this size. Low overall energy expenditure was mainly achieved in two ways. First, spotted antbirds had a very low locomotor activity during the day, compared with temperate-zone birds (M. Wikelski, K. S. Shel- don, and R. E. Ricklefs, unpublished manuscript). On average, spotted antbirds moved only 1.6 times/min and either hopped or flew only short distances. We cannot exclude that we missed some long flights (>20 m). However, our observation that spotted antbirds mostly hopped and never flew for more than 20 $\mathrm{m}$ at any one time is consistent with a previous study (Willis 1972). Spotted antbirds are facultative ant followers and take only $\sim 50 \%$ of their food at ant swarms (Willis 1972; M. Wikelski, behavioral observation). Thus, they exhibit short flights even if they are not roosting right over the bivouacs. Flight has been suggested to be one of the most expensive avian activities (Butler 1991); therefore, a low locomotor activity could be a way to save large amounts of energy. Measures of locomotor activity of free-living birds have not been standardized yet; thus, it is difficult to directly compare our estimate with those from other studies.

Second, spotted antbirds sleeping in the wild at $\sim 25^{\circ} \mathrm{C}\left(\sim 5^{\circ}-\right.$ $9^{\circ} \mathrm{C}$ below the TNZ) kept their nocturnal energy expenditure at levels similar to that of birds in the laboratory measured under thermoneutral conditions $\left(\sim 30^{\circ}-34^{\circ} \mathrm{C}\right.$ ambient temperature). How is this achieved? As inferred from laboratory respirometry trials, rest-phase thermoregulation in spotted antbirds was not consistent with typical avian patterns, that is, the maintenance of an approximately constant body temperature 
A

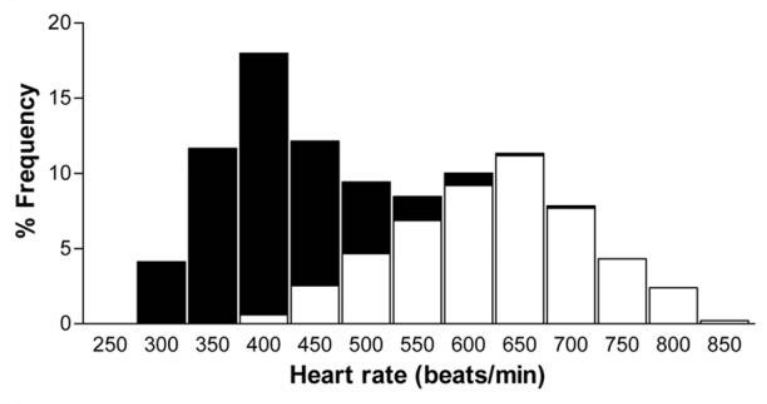

B

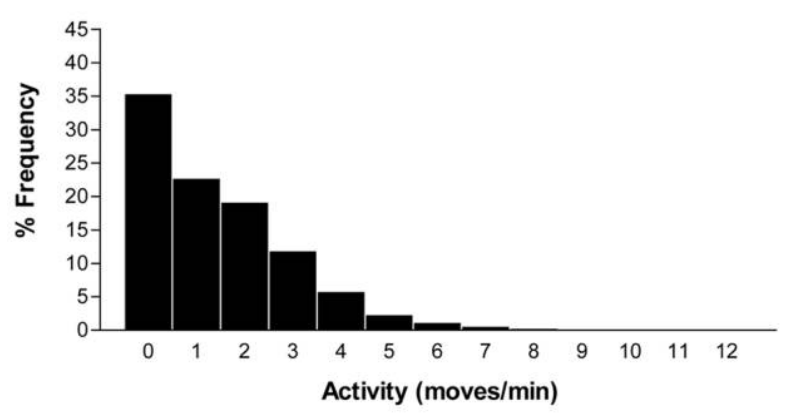

C

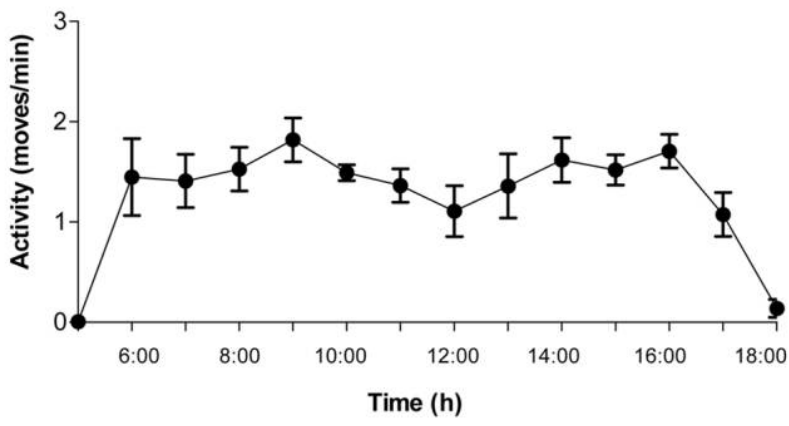

Figure 4. A, Bimodal frequency distribution of heart rate (beats/min) for six male spotted antbirds from Barro Colorado Island, Panama. Black bars represent heart rates measured at night $(N=725)$, and white bars indicate heart rate measured during the day $(N=730)$. Heart rate peaks occur at 400 and 650 beats/min. B, Frequency distribution of locomotor activity (hops and flights termed "moves") during the day (moves/min) for six male spotted antbirds from Barro Colorado Island. Data are highly right skewed, indicating generally low activity rates of wild spotted antbirds. C, Daily locomotor activity pattern (moves/min) for six male spotted antbirds from Barro Colorado Island. Data are hourly means $\pm 1 \mathrm{SE}$.

over a wide range of ambient temperatures (Blem 2000). Instead, spotted antbirds were able to markedly decrease their body temperature (Fig. 1). A decrease in body temperature reduces the difference between ambient temperature and body temperature and thus decreases the rate of heat loss. Hence, the demand for metabolic heat production decreases (MerolaZwartjes and Ligon 2000). We suggest that spotted antbirds use this strategy to lower metabolic rate at night. Similar instances of low body temperature (Bartholomew et al. 1983) or even torpor (sensu Geiser and Ruf 1995) have been found in a variety of bird species, such as the 6-g Puerto Rican tody (MerolaZwartjes and Ligon 2000), pigeons (Schleucher 2001), nightjars and nighthawks (McNab and Bonaccorso 1995), mousebirds (McKechnie et al. 2004), and white-throated sparrows (Dolby et al. 2004). However, while most studies only infer lowered metabolic activity from body temperature data, here we estimate it via Fick's equation from heart rate. Because the nutritional state of a bird is inversely correlated with the depth of nocturnal hypothermia (Reinertsen and Haftorn 1984), it is important to note that the captive experimental birds in this study were fed ad lib. and thus that the decrease of body tem-
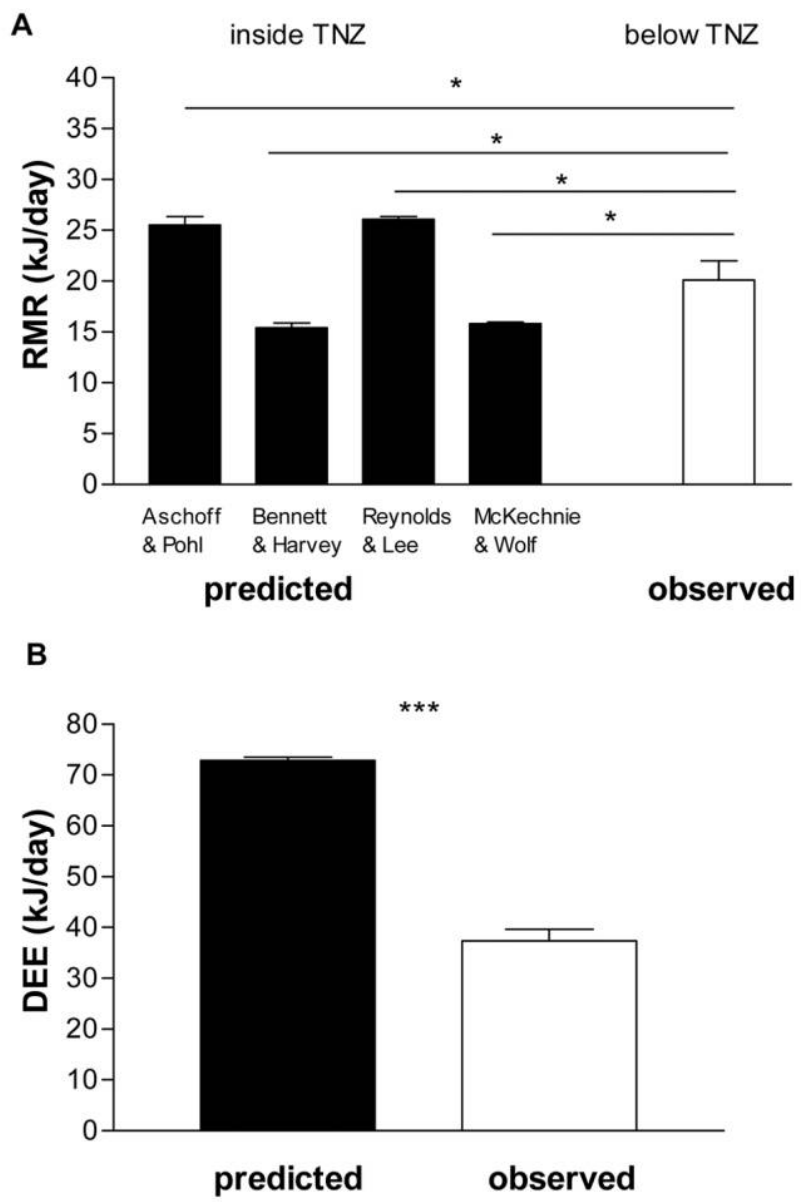

Figure 5. Predicted (black bars) and observed (via heart rate telemetry, white bars) resting metabolic rate (nighttime RMR, in $\mathrm{kJ} / \mathrm{d}$ ) $\pm 1 \mathrm{SE}$ $(A)$ and daily energy expenditure (DEE, in $\mathrm{kJ} / \mathrm{d}) \pm 1 \mathrm{SE}(B)$ for six male spotted antbirds. $A$, Predicted nighttime RMR values are from (i) the equation of Aschoff and Pohl (1970) for rest-phase passerines: $\log \mathrm{BMR}(\mathrm{kcal} / \mathrm{h})=\log 0.0317+0.726 \log m$, where $m=$ mass in grams; (ii) the equation of Bennett and Harvey (1987): log RMR $(\mathrm{kcal} / \mathrm{d})=0.67 \log m-0.25$; (iii) the phylogenetically corrected least squares regression of Reynolds and Lee (1996): $\log B M R(W)=$ $-1.311+0.635 \log m$; and (iv) the equation of McKechnie and Wolf (2004): $\log$ BMR (W) $=-1.461+0.669 \log m$. Horizontal bars represent pairwise comparisons between predicted and observed values; asterisk indicates $P<0.05$. $B$, Predicted DEE values are from the equation of Nagy et al. (1999) for small/temperate- forest birds: DEE $(\mathrm{kJ} / \mathrm{d})=15.94 m^{0.53}$, where $m=$ mass in grams. Three asterisks indicate $P<0.001$. 
perature was not caused by limited food supply. Therefore, as mentioned above, we hypothesize that the reduction of body temperature in spotted antbirds reflects a metabolic strategy for nocturnal energy saving. Two nonexclusive alternative explanations could account for the fact that estimated nighttime RMR in the field was below that measured for birds in a respirometer and within their TNZ. First, stress is expected to increase metabolic rates (Wingfield and Farner 1993). Thus, it is conceivable that birds in the laboratory did not completely settle in the respirometer. Second, measurement noise in the calibration (linear regression) between the metabolic rate and heart rate could have contributed to this result.

We conducted calibration experiments to determine the exact relationship between heart rate and oxygen consumption at a single ambient temperature. However, it has been shown that body temperature can affect the intercept of the relationship between oxygen consumption and heart rate (Butler et al. 2002). For example, in marine iguanas Amblyrhynchus cristatus, oxygen consumption was higher for a given level of heart rate as body temperature declined (Butler et al. 2002). Because the body temperature of free-living spotted antbirds may not have been constant, it is possible that the same effect may have occurred here. Because we did not measure heart rate in the birds for which we determined the relationship between ambient temperature, metabolic rate, and body temperature (Fig. 1 ), we were not able to adjust for this effect. Thus, we cannot exclude that RMR and DEE may have been underestimated. Ideally, the calibration of heart rate versus oxygen consumption should have been repeated at different ambient temperatures.

Nevertheless, spotted antbirds have life-history traits indicative of a slow pace of life (e.g., relatively long life spans, high annual survival rates, production of few offspring, slow development of offspring), similar to what has been observed in birds of paradise and other tropical bird groups (see Willis 1972, 1983; Brawn et al. 1995; Hau et al. 2000; Stutchbury and Morton 2001). Consistent with their slow pace of life, the metabolic rates - both nighttime RMR and DEE-of spotted antbirds are low. Because the decline in DEE is much higher than the decline in nighttime RMR, our data suggest that the low energy expenditure is predominantly caused by the decline in activity. Our data support and extend previous studies suggesting that life-history traits covary with the rate of energy expenditure. Future studies could investigate whether the low activity rates and low DEE are characteristic of this South American lineage of birds (antbirds; family Thamnophilidae).

\section{Acknowledgments}

We thank Peggy Han, Melissa Bowlin, Michaela Hau, Lynn B. Marty Martin, Kelly Lee, Holger Schielzeth, and Joe Williams for helpful comments and Rebecca Sealfon and Axel Haenssen for help with data processing. This work was supported by the National Science Foundation (grant IRCEB-IBN-0212587), the Smithsonian Tropical Research Institute, the Levinson Family Foundation, and Princeton University.

\section{Literature Cited}

Aschoff J. and H. Pohl. 1970. Der Ruheumsatz von Vögeln als Funktion der Tageszeit und der Körpergrösse. J Ornithol 111: 38-46.

Bartholomew G.A., C.M. Vleck, and T.L. Bucher. 1983. Energy metabolism and nocturnal hypothermia in two tropical passerine frugivores, Manacus vitellinus and Pipra mentalis. Physiol Zool 56:370-379.

Bartholomew G.A., D. Vleck, and C.M. Vleck. 1981. Instantaneous measurements of oxygen consumption during preflight warm-up and post-flight cooling in sphingid and saturniid moths. J Exp Biol 90:17-32.

Bennett P.M. and P.H. Harvey. 1987. Active and resting metabolism in birds: allometry, phylogeny and ecology. J Zool (Lond) 213:327-363.

Bevan R.M., J.R. Speakman, and P.J. Butler. 1995. Daily energy expenditure of tufted ducks: a comparison between indirect calorimetry, doubly labeled water and heart rate. Funct Ecol 9:40-47.

Bevan R.M., A.J. Woakes, P.J. Butler, and I.L. Boyd. 1994. The use of heart rate to estimate oxygen consumption of freeranging black-browed albatrosses Diomedea melanophrys. J Exp Biol 193:119-137.

Blem C.R. 2000. Energy balance. Pp. 327-341 in G.C. Whittow, ed. Sturkie's Avian Physiology. Academic Press, London.

Bowlin M.S. and M. Wikelski. 2008. Pointed wings, low wingloading and calm air reduce migratory flight costs in songbirds. PLoS ONE 3(5):e2154, doi:10.1371/journal.pone .0002154

Brawn J.D., J.R. Karr, and J.D. Nichols. 1995. Demography of birds in a Neotropical forest: effects of allometry, taxonomy, and ecology. Ecology 76:41-51.

Butler P.J. 1991. Exercise in birds. J Exp Biol 160:233-262.

Butler P.J., P.B. Frappell, T. Wang, and M. Wikelski. 2002. The relationship between heart rate and rate of oxygen consumption in Galapagos marine iguanas (Amblyrhynchus cristatus) at two different temperatures. J Exp Biol 205:1917-1924.

Butler P.J., J.A. Green, I.L. Boyd, and J.R. Speakman. 2004. Measuring metabolic rate in the field: the pros and cons of the doubly labelled water and heart rate methods. Funct Ecol 18:168-183.

Cochran W.W. and M. Wikelski. 2005. Individual migratory tactics of New World Catharus thrushes: current knowledge and future tracking options from space. Pp 274-289 in P. Marra and R. Greenberg, eds. Birds of Two Worlds: The Ecology and Evolution of Migration. Johns Hopkins University Press, Baltimore.

Cooke S.J., S.G. Hinch, M. Wikelski, R.D. Andrews, L.J. Kuchel, T.G. Wolcott, and P.J. Butler. 2004. Biotelemetry: a mechanistic approach to ecology. Trends Ecol Evol 19:334-343.

del Hoyo J., A. Elliot, and D.A. Christie. 2003. Handbook of the Birds of the World: Broadbills to Tapaculos. Vol. 8. Lynx, Barcelona.

Dietrich W., D. Windsor, and T. Dunne. 1982. Geology, hydrology and climate of Barro Colorado Island. Pp. 21-46 in 
J.E.G. Leigh, A.S. Rand, and D.M. Windsor, eds. The Ecology of a Tropical Forest: Seasonal Rhythms and Long-Term Changes. Smithsonian Institution Press, Washington, DC.

Dolby A.S., J.G. Temple, L.E. Williams, E.K. Dilger, K.M. Stechler, and V.S. Davis. 2004. Facultative rest-phase hypothermia in free-ranging white-throated sparrows. Condor 106:386-390.

Furness R.W. 2003. Metabolism: it's in the genes. Nature 425: 779-780.

Geiser F. and T. Ruf. 1995. Hibernation versus daily torpor in mammals and birds: physiological variables and classification of torpor patterns. Physiol Zool 68:935-966.

Green J.A., P.J. Butler, A.J. Woakes, and I.L. Boyd. 2002. Energy requirements of female macaroni penguins breeding at South Georgia. Funct Ecol 16:671-681.

Green J.A., P.J. Butler, A.J. Woakes, I.L. Boyd, and R.L. Holder. 2001. Heart rate and rate of oxygen consumption of exercising macaroni penguins. J Exp Biol 204:673-684.

Hails C.J. 1983. The metabolic rate of tropical birds. Condor 85:61-65.

Hamilton W.D. 1966. Moulding of senescence by natural selection. J Theor Biol 12:12-45.

Hau M., M. Wikelski, and J.C. Wingfield. 2000. Visual and nutritional food cues fine-tune timing of reproduction in a Neotropical rainforest bird. J Exp Zool 286:494-504.

Hawkins P.A.J., P.J. Butler, A.J. Woakes, and J.R. Speakman. 2000. Estimation of the rate of oxygen consumption of the common eider duck Somateria mollissima, with some measurements of heart rate during voluntary dives. J Exp Biol 203:2819-2832.

Klaassen M. 1995. Molt and basal metabolic costs in males of two subspecies of stonechats: the European Saxicola torquata rubicula and the East African S. t. axillaris. Oecologia 104: 424-432.

Lindstrom A. and M. Klaassen. 2003. High basal metabolic rates of shorebirds while in the Arctic: a circumpolar view. Condor 105:420-427.

Martin T.E. 1996. Life history evolution in tropical and south temperate birds: what do we really know? J Avian Biol 27: 263-272.

McKechnie A.E., G. Körtner, and B.G. Lovegrove. 2004. Restphase thermoregulation in free-ranging white-backed mousebirds. Condor 106:143-149.

McKechnie A.E. and B.O. Wolf. 2004. The allometry of avian basal metabolic rate: good predictions need good data. Physiol Biochem Zool 77:502-521.

McNab B.K. and F.J. Bonaccorso. 1995. The energetics of Australasian swifts, frogmouths, and nightjars. Physiol Zool 68: 245-261.

Merola-Zwartjes M. and J.D. Ligon. 2000. Ecological energetics of the Puerto Rican tody: heterothermy, torpor, and intraisland variation. Ecology 81:990-1003.

Nagy K.A., I.A. Girard, and T.K. Brown. 1999. Energetics of free-ranging mammals, reptiles, and birds. Annu Rev Nutr 19:247-277.

Nolet B.A., P.J. Butler, D. Masman, and A.J. Woakes. 1992. Estimation of daily energy expenditure from heart rate and doubly labeled water in exercising geese. Physiol Zool 65: 1188-1216.

Raim A. 1978. A radio transmitter attachment for small passerine birds. Bird-Banding 49:326-332.

Reinertsen R.E. and S. Haftorn. 1984. The effect of short-time fasting on metabolism and nocturnal hypothermia in the willow tit Parus montanus. J Comp Physiol 154:23-28.

Reynolds P.S. and R.M. Lee. 1996. Phylogenetic analysis of avian energetics: passerines and nonpasserines do not differ. Am Nat 147:735-759.

Ricklefs R.E. 1991. Structures and transformations of life histories. Funct Ecol 5:174-183.

Ricklefs R.E., M. Konarzewski, and S. Daan. 1996. The relationship between basal metabolic rate and daily energy expenditure in birds and mammals. Am Nat 147:1047-1071.

Ricklefs R.E. and J.B. Williams. 2003. Metabolic responses of shorebird chicks to cold stress: hysteresis of cooling and warming phases. J Exp Biol 206:2883-2893.

Ridgely R. and J. Gwynne. 1989. A Guide to the Birds of Panama. 2nd ed. Princeton University Press, Princeton, NJ.

Schleucher E. 2001. Heterothermia in pigeons and doves reduces energetic costs. J Therm Biol 26:287-293.

Shaffer S.A., D.P. Costa, and H. Weimerskirch. 2001. Comparison of methods for evaluating energy expenditure of incubating wandering albatrosses. Physiol Biochem Zool 74: 823-831.

Speakman J.R. 1998. The history and theory of the doubly labeled water technique. Am J Clin Nutr 68:932S-938S.

Stutchbury B.J.M. and E.S. Morton. 2001. Behavioral Ecology of Tropical Birds. Academic Press, London.

Tieleman B.I. 2007. Differences in the physiological responses to temperature among stonechats from three populations reared in a common environment. Comp Biochem Physiol A 146:194-199.

Tieleman B.I., T.H. Dijkstra, J.R. Lasky, R.A. Mauck, G.H. Visser, and J.B. Williams. 2006. Physiological and behavioural correlates of life-history variation: a comparison between tropical and temperate zone house wrens. Funct Ecol 20: 491-499.

Vleck C.M. and D. Vleck. 1979. Metabolic rate in five tropical bird species. Condor 81:89-91.

Walsberg G.E. and T.C.M. Hoffman. 2005. Direct calorimetry reveals large errors in respirometric estimates of energy expenditure. J Exp Biol 208:1035-1043.

Walsberg G.E. and B.O. Wolf. 1995. Variation in the respiratory quotient of birds and implications for indirect calorimetry using measurements of carbon dioxide production. J Exp Biol 198:213-219.

Weathers W.W. 1997. Energetics and theromoregulation by small passerines of the humid, lowland tropics. Auk 114: 341-353.

Weathers W.W. and F.G. Stiles. 1989. Energetics and water balance in free-living tropical hummingbirds. Condor 91:324331.

Wiersma P., A. Muñoz-Garcia, A. Walker, and J.B. Williams. 
2007. Tropical birds have a slow pace of life. Proc Natl Acad Sci USA 104:9340-9345.

Wikelski M., M. Hau, and J.C. Wingfield. 1999. Social instability increases plasma testosterone in a year-round territorial Neotropical bird. Proc R Soc B 266:551-556.

Wikelski M. and R.E. Ricklefs. 2001. The physiology of life histories. Trends Ecol Evol 16:479-481.

Wikelski M., L. Spinney, W. Schelsky, A. Scheuerlein, and E. Gwinner. 2003. Slow pace of life in tropical sedentary birds: a common-garden experiment on four stonechat populations from different latitudes. Proc R Soc B 270:2383-2388.

Willis E.O. 1972. The behavior of spotted antbirds. Ornithol Monogr 10:1-157.
1983. Longevities of some Panamanian forest birds, with note of low survivorship in old spotted antbirds $\mathrm{Hy}$ lophylax naevioides. J Field Ornithol 54:413-414.

Wingfield J.C. and D.S. Farner. 1993. Endocrinology of reproduction in wild species. Pp. 163-327 in D.S. Farner, J.R. King, and K.C. Parkes, eds. Avian Biology. Vol. 9. Harcourt Brace, London.

Withers P.C. 1977. Measurement of $\dot{\mathrm{V}}_{2}, \dot{\mathrm{V}}_{\mathrm{CO}_{2}}$, and evaporative water loss with a flow-through mask. J Appl Physiol 42:120 123.

2001. Design, calibration and calculation for flowthrough respirometry systems. Aust J Zool 49:445-461. 